\title{
A MODEL FOR THE EFFECT OF ROUGHNESS ON THE LOAD CAPACITY OF JOURNAL BEARINGS
}

\author{
BY \\ D. W. PRAVICA
}

Dept. of Mathematics, East Carolina University, Greenville, North Carolina

\begin{abstract}
The flow generated by a rotating circular cylinder inside a corrugated outer cylinder is considered. The corrugations are small and are used to model roughness. The force on the inner cylinder, as a linear function of displacement, is found. This requires a Stokes expansion to determine a first-order correction in the Reynolds number $R$. The result implies as expected that transverse roughness acts to increase the load capacity of the bearing at low $R$, which is confirmed by previous numerical experiments. In addition, roughness can induce reversed flow at much smaller displacements than for smooth bearings.
\end{abstract}

1. Introduction. There are a number of classical problems in fluid dynamics which, due to their geometric simplicity, yield much information to mathematical analysis. The study of two-dimensional flow of a viscous fluid between rotating cylinders is one such example. Most works have considered the case of eccentric cylinders, where spacing between the walls is small (i.e., thin film). Perturbation theory on the Stokes equation, ignoring curvature terms, leads to the Reynolds equation. The latter equation has been studied, in its stochastic form [5], with the objective of understanding the effect of roughness on load capacity of plane bearings. However, in order to obtain a good model for journal bearings, most authors have used the bipolar coordinate transformation. DiPrima and Stuart have considered the inclusion of inertial effects, by using a Stokes expansion of the steady state Navier-Stokes equation [2]. A comprehensive study of streamline patterns, when both cylinders are in rotation, can be found in [1]. Most recent work has concentrated on numerical schemes, which include time-dependent perturbations, extensions of the stochastic model of roughness, and turbulent flows [4].

The purpose of this paper is to extend the classical approach to the above stated problem (e.g. [8]). It is based on work initiated by K. B. Ranger [6]. In [6] the (generalized) fluted column transformation is defined and is used to solve for the creeping flow $(R=0)$ between a fixed spatially periodic outer boundary and a smooth rotating inner boundary. An extension to this solution is found in [3], where a linearized

Received March 9, 1993.

1991 Mathematics Subject Classification. Primary 76D20.

This work was supported in part by a grant from NSERC.

(C)1995 Brown University 
convection (or inertial) assumption to the Navier-Stokes equation is considered. It is found that the torque exerted on the inner cylinder increases with Reynolds number; however, this is only observed after obtaining a quadratic correction to the convection. In this paper, the normal forces on the inner cylinder are obtained for small eccentricity (i.e., off-centre parameter). The torque remains constant. The angular velocity of the inner cylinder changes with high-order corrections.

The paper is organized as follows. In Sec. 2 the problem is stated and the special coordinates are introduced. Section 3 considers a perturbation in the eccentricity parameter. This involves the construction of biharmonic corrections to the original stream function found in [6]. A linearized inertial effect on the solution is considered in Sec. 4, which extends the work in [3] to the case of off-centre cylinders. For brevity only the thin film results are given. Section 5 is a discussion of the perturbation solution. By examining boundary vorticity it is found that separation occurs as a consequence of roughness. The paper is completed in Sec. 6 with a derivation of the force components and a comment on changes in load capacity and stability.

2. The flow problem. Consider the situation of a stationary sleeve of varying radius $(1 \pm \varepsilon)$, containing a cylinder of radius $\lambda(<1-\varepsilon)$. The number of crevices on the outer boundary is taken to be large (i.e., $n \gg 1)$. In general, the cylinder centres will differ by a small value $\delta$ (see Fig. 1). We make use of the following parameters:

$$
\begin{array}{ll}
\varepsilon: \text { roughness, } & 1-\lambda: \text { mean clearance, } \\
\delta: \text { journal displacement, } & 2 \pi / n: \text { roughness wavelength } .
\end{array}
$$

It is assumed that

$$
\begin{gathered}
\varepsilon<(1-\lambda), \quad \lambda^{n} \ll 1, \quad 0 \leq m \equiv \varepsilon(n+1)<1, \\
(1-\lambda-\varepsilon)>\delta \gg \delta^{2} \gg \delta / n \gg \delta^{3} \gg \delta^{2} / n .
\end{gathered}
$$

Terms of order $\delta / n$ are retained, but terms of order $\delta^{3}$ and $\lambda^{n}$ are ignored. The thin film approximation, in the presence of corrugations, corresponds to

$$
\begin{gathered}
m \lambda^{n} \ll 1, \quad m \ll 1, \\
1 \gg \frac{m \varepsilon}{1-\lambda^{2}} \gg\left(1-\lambda^{2}\right) .
\end{gathered}
$$

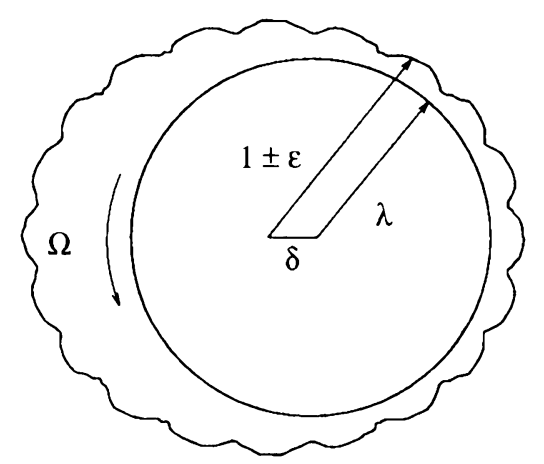

FIG. 1. Definition of region. Fluid area is $\pi\left(1+m \varepsilon-\lambda^{2}\right)$. 
Here, terms of order $m \lambda^{n}$ and $m^{2} \varepsilon$ are rejected. For the expansion in the Reynolds number $R$, terms of order $\delta R m \varepsilon$ are retained in the stream function.

The conformal mapping of interest is the fluted column transformation

$$
z=\zeta+\varepsilon \zeta^{n+1}, \quad z=x+i y, \quad \zeta=\rho e^{i \phi},
$$

which is conformal in $|\zeta| \leq 1$ for $m<1$ (i.e., the mapping factor is positive, $|d z / d \zeta|^{2}>0$ ). The Laplacian in the $z$-plane and in the $\zeta$-plane are related by (from [6])

$$
\begin{aligned}
\nabla^{2} & =\frac{\partial^{2}}{\partial x^{2}}+\frac{\partial^{2}}{\partial y^{2}}=\left|\frac{d z}{d \zeta}\right|^{-2} \nabla_{\rho}^{2}, \\
\nabla_{\rho}^{2} & =\frac{\partial^{2}}{\partial \rho^{2}}+\frac{1}{\rho} \frac{\partial}{\partial \rho}+\frac{1}{\rho^{2}} \frac{\partial^{2}}{\partial \phi^{2}}, \\
\left|\frac{d z}{d \zeta}\right|^{2} & =1+2 m \rho^{n} \cos n \phi+m^{2} \rho^{2 n}, \\
\left|\frac{d z}{d \zeta}\right|^{-2} & =\frac{1}{1-m^{2} \rho^{2 n}}\left(1+2 \sum_{k=1}^{\infty}\left(-m \rho^{n}\right)^{k} \cos k n \phi\right) .
\end{aligned}
$$

The fluid is considered incompressible; hence, $\vec{q}$ can be written in terms of a stream function, $\psi(x, y)$, as follows:

$$
\vec{q}=(u, v)=\left(-\partial_{y} \psi, \partial_{x} \psi\right) .
$$

The Navier-Stokes equation for steady flow then reduces to the vorticity equation,

$$
\nabla^{2} \omega=R \frac{\partial\left(\psi, \nabla^{2} \psi\right)}{\partial(x, y)}=R\left(\partial_{x} \psi \partial_{y} \omega-\partial_{y} \psi \partial_{x} \omega\right),
$$

where the vorticity and Reynolds number are, respectively,

$$
\omega=\nabla^{2} \psi, \quad R=1 / \nu .
$$

One concern of this paper is with the effect of linearized convection. Hence, the stream function is assumed to take the form

$$
\psi(x, y)=\psi_{0}(x, y)+R \psi_{1}(x, y),
$$

and the Navier-Stokes equation reduces to

$$
\begin{aligned}
& \nabla^{4} \psi_{0}(x, y)=0, \\
& \nabla^{4} \psi_{1}(x, y)=\frac{\partial\left(\psi_{0}, \nabla^{2} \psi_{0}\right)}{\partial(x, y)} .
\end{aligned}
$$

The boundary conditions (b.c.) for the case of concentric cylinders $\delta=0$ ), where the inner one $(\rho=\lambda)$ rotates with angular velocity $\Omega$, are $\left(c_{i}\right.$ cr.stants $)$

$$
\begin{array}{llll}
\left.\psi_{0}\right|_{\rho=1}=c_{1}, & \left.\partial_{\rho} \psi_{0}\right|_{\rho=1}=0, & \left.\psi_{0}\right|_{\rho=\lambda}=c_{2}, & \left.\partial_{\rho} \psi_{0}\right|_{\rho=\lambda}=\Omega \lambda \\
\left.\psi_{1}\right|_{\rho=1}=c_{3}, & \left.\partial_{\rho} \psi_{1}\right|_{\rho=1}=0, & \left.\psi_{1}\right|_{\rho=\lambda}=c_{4}, & \left.\partial_{\rho} \psi_{1}\right|_{\rho=\lambda}=0 .
\end{array}
$$


The stream function and vorticity for the Stokes equation $(2.6 \mathrm{~b})$ are $\left(\lambda^{n} \ll 1\right)$

$$
\begin{aligned}
\psi_{0} & =\mathscr{A}\left[\log \rho-\frac{\left(\rho^{2}-\varepsilon^{2} \rho^{2 n+2}\right)}{2(1-m \varepsilon)}\right], \quad \mathscr{A}=\frac{\Omega \lambda^{2}(1-m \varepsilon)}{\left(1-m \varepsilon-\lambda^{2}\right)}, \\
\nabla^{2} \psi_{0} & =-2 \mathscr{A}^{\prime}\left[1+2 \sum_{k=1}^{\infty}\left(-m \rho^{n}\right)^{k} \cos k n \phi\right], \quad \mathscr{A}^{\prime}=\mathscr{A} /(1-m \varepsilon) .
\end{aligned}
$$

(See [6].) The first-order correction, $\psi_{1}$, is obtained in [3] and will not be given here.

3. Perturbation in $\delta$. The cylinders shall no longer be considered concentric. Let the inner cylinder be shifted to the right by a distance $\delta$. It is convenient to introduce the polar coordinate system $\left(\rho_{\delta}, \phi_{\delta}\right)$ which has as origin the centre of the inner cylinder. Then

$$
\begin{aligned}
\rho_{\delta}^{2} & =\rho^{2}-2 \delta \rho \cos \phi+\delta^{2}, \\
\rho^{2} & =\rho_{\delta}^{2}+2 \delta \rho_{\delta} \cos \phi_{\delta}+\delta^{2}, \\
\rho_{\delta} \sin \phi_{\delta} & =\rho \sin \phi, \quad \rho_{\delta} \cos \phi_{\delta}=\rho \cos \phi, \\
\nabla_{\rho}^{2} & =\nabla_{\rho_{\delta}}^{2} .
\end{aligned}
$$

A new biharmonic solution results:

$$
\begin{aligned}
& \psi_{0}^{\delta}=\Psi_{0}(\delta)+\delta \Phi_{1}+\delta^{2} \Phi_{2}+O\left(\delta^{3}\right), \\
& \psi_{1}^{\delta}=\Psi_{1}(\delta)+\delta \Lambda_{1}+\delta^{2} \Lambda_{2}+O\left(\delta^{3}\right) .
\end{aligned}
$$

The perturbation analysis begins by choosing

$$
\Psi_{0}(\delta)=\mathscr{A}^{\prime}\left[(1-m \varepsilon) \log \rho_{\delta}-\frac{1}{2}\left(\rho_{\delta}^{2}-\varepsilon^{2} \rho^{2 n+2}\right)\right] .
$$

Clearly, $\Psi_{0}(\delta)$ satisfies the inner b.c. (i.e., as $\rho_{\delta} \rightarrow \lambda$ ) to order $\lambda^{n}$. The function $\Phi_{1}$ is a biharmonic correction to the outer b.c., which is obtained by expanding $\Psi_{0}(\delta)$ at $\rho=1$,

$$
\begin{gathered}
\Psi_{0}(\delta)(\rho=1)=\delta \mathscr{A}^{\prime} m \varepsilon \cos \phi-\delta^{2} \frac{\mathscr{A}}{2} \cos 2 \phi+c_{5}+O\left(\delta^{3}\right), \\
\partial_{\rho} \Psi_{0}(\delta)(\rho=1)=\delta \mathscr{A}^{\prime}(2-m \varepsilon) \cos \phi+\delta^{2} \mathscr{A} \cos 2 \phi+O\left(\delta^{3}\right) .
\end{gathered}
$$

The correction $\Phi_{2}$ depends on the inner boundary values of $\Phi_{1}$ and the outer boundary values of $\Psi_{0}(\delta)$. A standard method of constructing biharmonic functions is to write

$$
\begin{aligned}
\chi_{\alpha}(\rho) & =x \rho^{\alpha} \cos \alpha \phi+y \rho^{\alpha} \sin \alpha \phi \\
& =\rho^{\alpha+1} \cos (\alpha-1) \phi+\varepsilon \rho^{\alpha+n+1} \cos (\alpha-(n+1)) \phi .
\end{aligned}
$$

Then, from (3.2), (3.4), and (2.7), the appropriate corrections are chosen to be

$$
\begin{aligned}
\Phi_{1}= & A \chi_{2}+B \chi_{n}+C \chi_{n+2}+D \log \rho \chi_{0} \\
& +(E \rho+F / \rho) \cos \phi+G \rho^{n-1} \cos (n-1) \phi+H \rho^{n+1} \cos (n+1) \phi \\
\Phi_{2}= & A^{\prime} \chi_{3}+B^{\prime} \chi_{n-1}+C^{\prime} \chi_{n+3}+D^{\prime} \chi_{-1} \\
& +\left(E^{\prime} \rho^{2}+F^{\prime} / \rho^{2}\right) \cos 2 \phi+G^{\prime} \rho^{n-2} \cos (n-2) \phi+H^{\prime} \rho^{n+2} \cos (n+2) \phi
\end{aligned}
$$


and the coefficients should satisfy the b.c.

$$
\begin{aligned}
& \rho=1, \quad \Phi_{1}=-m \varepsilon \mathscr{A}^{\prime} \cos \phi, \quad \partial_{\rho} \Phi_{1}=(m \varepsilon-2) \mathscr{A}^{\prime} \cos \phi, \\
& \rho_{\delta}=\lambda, \quad \Phi_{1}=\partial_{\rho} \Phi_{1}=0, \\
& \rho=1, \quad \Phi_{2}=\frac{1}{2} \mathscr{A} \cos 2 \phi, \quad \partial_{\rho} \Phi_{2}=-\mathscr{A} \cos 2 \phi, \\
& \rho_{\delta}=\lambda, \quad \Phi_{2}=0, \quad \partial_{\rho} \Phi_{2}=-\left(4 A \lambda^{2}+D\right) \lambda^{-1}\{1+\cos 2 \phi\} .
\end{aligned}
$$

The first-order correction is found to be (to order $1 / n^{2}$ ),

$$
\begin{aligned}
\Phi_{1}= & {\left[A \rho^{2}\left(1-(\lambda / \rho)^{2}\right)^{2}-D \frac{1}{2}\left(2 \log (\lambda / \rho)+1-(\lambda / \rho)^{2}\right)-\frac{\varepsilon^{2}}{2} \rho^{2 n}\left(4 A+D \rho^{2}\right)\right] \rho \cos \phi } \\
& +A \varepsilon\left(\rho^{2}-1\right)^{2} \rho^{n-1} \cos (n-1) \phi+D \frac{\varepsilon}{2}\left(2 \log \rho+1-\rho^{2}\right) \rho^{n+1} \cos (n+1) \phi \\
A= & \frac{-2 \mathscr{A}^{\prime}+D(m \varepsilon+2 \log \lambda)}{4\left(1-m \varepsilon-\lambda^{2}\right)}, \\
D= & -\mathscr{A}^{\prime} \frac{\left(1-\lambda^{2}\right)-2 m \varepsilon}{\left(1-\lambda^{2}\right)+\left(1+\lambda^{2}\right) \log \lambda-2 m \varepsilon\left[(\log \lambda) /\left(1-\lambda^{2}\right)+1-\frac{1}{4}\left(1+\lambda^{2}\right)\right]}
\end{aligned}
$$

For $\Phi_{2}$ the b.c. (3.7b) cannot be satisfied using a solution of the form (3.6b). Instead, we use the inner b.c.

$$
\partial_{\rho} \Phi_{2}(\lambda)=-\left(4 A \lambda^{2}+D\right) \lambda^{-1} \cos 2 \phi,
$$

which changes the angular velocity of the rotating cylinder, to order $\delta^{2}$. This gives

$$
\begin{gathered}
\Phi_{2}=\frac{1}{2}\left[A^{\prime} \rho^{4}\left(2-3(\lambda / \rho)^{2}+(\lambda / \rho)^{6}\right)-D^{\prime}(\rho / \lambda)^{2}\left(1-(\lambda / \rho)^{2}\right)^{2}\right. \\
+\varepsilon A^{\prime}\left(2+\rho^{2}\right)\left(1-\rho^{2}\right)^{2} \rho^{n-2} \cos (n-2) \phi+\varepsilon D^{\prime}\left(1-\rho^{2}\right)^{2} \rho^{n} \cos (n+2) \phi \\
4 A \lambda^{2}+D=-\mathscr{A} \frac{\left(1-\lambda^{4}\right)+4 \lambda^{4} \log \lambda}{\left(1-\lambda^{2}\right)\left(1-\lambda^{2}+\left(1+\lambda^{2}\right) \log \lambda\right)}+O(m \varepsilon) \\
A^{\prime}=-\frac{\mathscr{A}}{\left(1-\lambda^{2}\right)^{3}}-\frac{4 A \lambda^{2}+D}{2\left(1-\lambda^{2}\right)^{2}} \\
D^{\prime}=3 A^{\prime} \frac{\lambda^{2}}{\left(1-\lambda^{2}\right)^{3}}-\frac{4 A \lambda^{2}+D}{2\left(1-\lambda^{2}\right)}\left(1+2 \lambda^{2}\right) .
\end{gathered}
$$

4. Linearized convection. The computations in this section are performed using Mathematica; so the discussion will be brief. Results will be given for the thin film approximation (i.e., leading-order terms in $(1-\lambda)$ ). This corresponds to ignoring wall curvature effects. Full expressions are easily obtained, but are not presented.

The linear Stokes correction term, $\psi_{1}^{\delta}$, is defined in $(3.2 \mathrm{~b})$. The zeroth-order correction (in $\delta$ ) is found by satisfying

$$
\nabla^{4} \Psi_{1}(\delta)=\partial\left(\Psi_{0}(\delta), \nabla^{2} \Psi_{0}(\delta)\right) / \partial(x, y),
$$


and the result is given in [3]. The solution is a Fourier series in $\{\sin (k n \phi)\}_{k=1}^{\infty}$, and of order $\lambda^{n}$ near the inner cylinder. For $n \gg 1$ it gives no contribution to the force (see Sec. 6); so there is no need to consider this term further.

The first-order correction (in $\delta$ ) satisfies

$$
\nabla_{\rho}^{2}\left(\nabla^{2} \Lambda_{1}\right)=\frac{1}{\rho} \partial\left(\Psi_{0}(\delta), \nabla^{2} \Phi_{1}\right) / \partial(\rho, \phi)+\frac{1}{\rho} \partial\left(\Phi_{1}, \nabla^{2} \Psi_{0}(\delta)\right) / \partial(\rho, \phi) .
$$

Now the solution can be partitioned as

$$
\Lambda_{1}=\Lambda_{1}^{p}+\Lambda_{1}^{b h}
$$

where $\Lambda_{1}^{p}$ is a particular solution of (4.2) and $\lambda_{1}^{b h}$ is a biharmonic correction to the resulting b.c. The particular solution is found by first assuming it to satisfy

$$
\nabla^{2} \Lambda_{1}^{p}=\sum_{k=1}^{\infty} A_{k}(\rho) \sin k \phi, \quad \Lambda_{1}^{p}=\sum_{k=1}^{\infty} B_{k}(\rho) \sin k \phi .
$$

Equation (4.2) then reduces to an Euler equation for the $A_{k}$ 's. Hence, integrating term by term gives

$$
\left|\frac{d z}{d \zeta}\right|^{-2} \nabla_{\rho}^{2} \Lambda_{1}^{p}=A_{1} \sin \phi+\sum_{l=1}^{\infty} A_{l n \pm 1} \sin (\ln \pm 1) \phi .
$$

The coefficients consist of finite polynomials of $\rho$ and $\log (\rho)$ terms. Multiplying through by the mapping factor $(2.3 \mathrm{c})$ and integrating $(4.4 \mathrm{~b})$ once more gives

$$
\Lambda_{1}^{p}=B_{1} \sin \phi+B_{n \pm 1} \sin (n \pm 1) \phi+B_{2 n \pm 1} \sin (2 n \pm 1) \phi+O\left(m^{2} \varepsilon ; 1 / n^{2}\right) .
$$

This is the particular solution. The coefficient $B_{1}(\rho)$ is given in the appendix.

A biharmonic correction must be a Fourier series in the five sin functions of (4.5). Hence, define

$$
\begin{aligned}
\bar{\chi}_{\alpha}(\rho) & =x \rho^{\alpha} \sin \alpha \phi-y \rho^{\alpha} \cos \alpha \phi \\
& =\rho^{\alpha+1} \sin (\alpha-1) \phi+\varepsilon \rho^{\alpha+n+1} \sin (\alpha-(n+1)) \phi,
\end{aligned}
$$

and set

$$
\begin{aligned}
\Lambda_{1}^{b h}= & \bar{A} \bar{\chi}_{2}+\bar{B} \bar{\chi}_{n}+\bar{C} \bar{\chi}_{n+2}+\bar{D} \log \rho \bar{\chi}_{0} \\
& +(\bar{E} \rho+\bar{F} / \rho) \sin \phi+\bar{G} \rho^{n-1} \sin (n-1) \phi+\bar{H} \rho^{n+1} \sin (n+1) \phi \\
& +\bar{J} \bar{\chi}_{2 n}+\bar{K} \bar{\chi}_{2 n+2}+\bar{L} \rho^{2 n-1} \sin (2 n-1) \phi+\bar{M} \rho^{2 n+1} \sin (2 n+1) \phi .
\end{aligned}
$$

The coefficients are found by satisfying the b.c. implied by $(3.2 b)$, i.e.,

$$
\begin{aligned}
& \left.\Lambda_{1}^{b h}\right|_{\rho=1}=-\left.\Lambda_{1}^{p}\right|_{\rho=1},\left.\quad \partial_{\rho} \Lambda_{1}^{b h}\right|_{\rho=1}=-\left.\partial_{\rho} \Lambda_{1}^{p}\right|_{\rho=1}, \\
& \left.\Lambda_{1}^{b h}\right|_{\rho_{\delta}=\lambda}=-\left.\Lambda_{1}^{p}\right|_{\rho_{\delta}=\lambda},\left.\quad \partial_{\rho_{\delta}} \Lambda_{1}^{b h}\right|_{\rho_{\delta}=\lambda}=-\left.\partial_{\rho_{\delta}} \Lambda_{1}^{p}\right|_{\rho_{\delta}=\lambda} \text {. }
\end{aligned}
$$

In particular, $\bar{D}$ and $\bar{A}-\bar{F} / \lambda^{4}$ are given in the appendix, under condition (2.1d). This is the only information needed to determine the force components. Applying the same procedure for $\Lambda_{2}$ leads to a solution in terms of the functions $\{\sin (k n \pm$ 2) $\}_{k=-\infty}^{\infty}$. This will not contribute to the force to $O\left(\delta^{3}\right)$. 
5. Separation of streamlines. To study boundary separation it is natural to consider the vorticity. In this section Stokes flow $(R=0)$ is examined under the conditions (2.1a) and (2.1b). Since $\delta>0$ this extends the work of [6].

The vorticity is written as

$$
\omega^{\delta}=\nabla^{2} \psi_{0}^{\delta}=|d z / d \zeta|^{2} \nabla_{\rho}^{2} \psi_{0}^{\delta} .
$$

Define $W(\rho, \phi)=|d z / d \zeta|^{2} \omega\left(\rho_{\delta}, \phi_{\delta}\right)$. Then, using Eqs. (3.2a), (3.8a), and (3.9a) with the formulas $(\alpha \neq 0)$

$$
\begin{aligned}
\nabla_{\rho}^{2} \chi_{\alpha}(\rho) & =4 \alpha\left(\rho^{\alpha-1} \cos (\alpha-1) \phi+m \rho^{n+\alpha-1} \cos (\alpha-n-1) \phi\right), \\
\nabla_{\rho}^{2} x \log \rho & =2\left(\rho^{-1} \cos \phi+m \rho^{n-1} \cos (n+1) \phi\right),
\end{aligned}
$$

gives on the outer boundary

$$
\begin{aligned}
W(\rho=1, \phi)= & -2 \mathscr{A}^{\prime}\left(1-m^{2}\right)+\delta[2(4 A(1-m \varepsilon n)+D(1-m \varepsilon(n+2))) \cos \phi \\
& +8 A \varepsilon \cos (n-1) \phi-2 D \varepsilon \cos (n+1) \phi] \\
& +\delta^{2}\left[4\left(1-m^{2}\right)\left(3 A^{\prime}-D^{\prime}\right) \cos 2 \phi\right]+O\left(1 / n^{2}, \delta^{2} / n, \delta^{3}\right) .
\end{aligned}
$$

If separation occurs on the boundary, it will begin in the neighbourhood of $\phi=\pi$ (i.e., point furthest from inner boundary when $\delta>0$ ). Then computing, for $\varepsilon>0$ and $n$ even,

$$
\begin{array}{r}
W(\phi=(1 \pm 1) \pi / 2)=-2\left\{\left(\mathscr{A}^{\prime}-\delta(4 A+D)+2 \delta^{2}\left(3 A^{\prime}-D^{\prime}\right)\right)\left(1-m^{2}\right)\right. \\
\pm 4 \delta m \varepsilon(4 A-D)\}+O\left(1 / n^{2}, \delta^{2} / n, \delta^{3}\right) .
\end{array}
$$

It is clear that if $\delta>0$, then for some $m$ sufficiently near 1 , separation occurs (i.e., $\omega^{\delta}$ changes sign). The extent of separation will be of the order of the roughness width $1 / n$, as indicated by Figs. 2 and 3. As the Reynolds number increases, so will the extent of separation and the effect of roughness on the flow [3]. Physically this is important because the onset of separation in theory usually indicates turbulent behaviour in practice. Turbulence in journal bearings reduces stability and increases heat production [4]. This is an undesirable aspect of boundary perturbations.

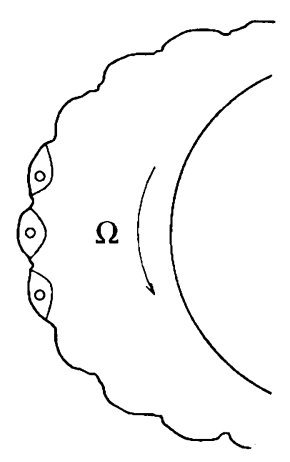

FIG. 2. Separation in crevices for even $n$ case 


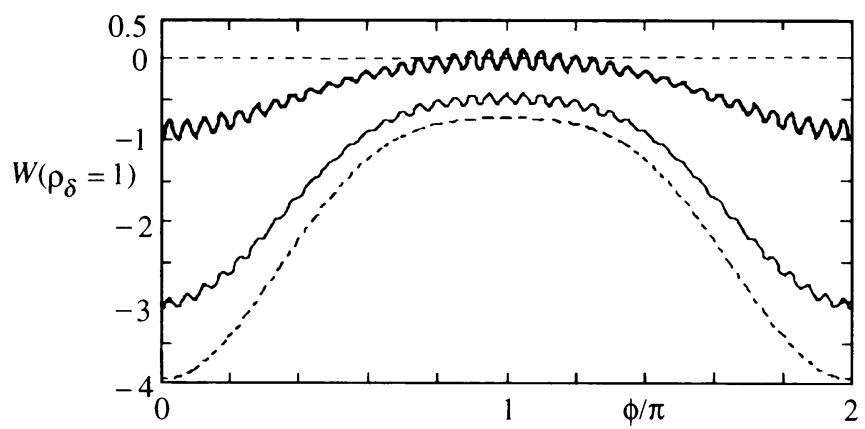

Fig. 3. Boundary vorticity for $\lambda=0.6, \delta=0.08, n=40$, and $m=0.0,0.5,0.9$

6. Force on the inner cylinder. Recall the well-known force and torque formulas [7],

$$
\begin{aligned}
& \mathscr{F}_{x}=\mu \lambda^{2} \int_{0}^{2 \pi}\left(\partial_{\rho_{\delta}}^{3} \psi\right)_{\rho_{\delta}=\lambda} \sin \phi_{\delta} d \phi_{\delta}, \\
& \mathscr{F}_{y}=-\mu \lambda^{2} \int_{0}^{2 \pi}\left(\partial_{\rho_{\delta}}^{3} \psi\right)_{\rho_{\delta}=\lambda} \cos \phi_{\delta} d \phi_{\delta}, \\
& \mathscr{G}=\mu \lambda^{2} \int_{0}^{2 \pi}\left(\partial_{\rho_{\delta}}^{2} \psi-\lambda^{-1} \partial_{\rho_{\delta}} \psi\right)_{\rho_{\delta}=\lambda} d \phi_{\delta} .
\end{aligned}
$$

The coordinate transformation implies the relations

$$
\begin{aligned}
\partial \rho / \partial \rho_{\delta} & =1+O\left(\delta^{2}\right), \\
\frac{\partial}{\partial \phi} \sin \phi_{\delta} & =\cos \phi+\delta \cos 2 \phi+O\left(\delta^{2}\right), \\
\frac{\partial}{\partial \phi} \cos \phi_{\delta} & =-\sin \phi+\delta \sin 2 \phi+O\left(\delta^{2}\right) .
\end{aligned}
$$

Upon substituting the results of the previous sections, the forces are, to $O\left(\delta^{3}\right)$,

$$
\begin{gathered}
\mathscr{F}_{x}^{\varepsilon}=\mu \lambda^{2} \int_{0}^{2 \pi}\left(\partial_{\rho}^{3} \delta \Lambda_{1}\right)_{\rho=\lambda} \sin \phi d \phi \\
=\mu \lambda^{2} \pi \delta\left[\left(\partial_{\rho}^{3} B_{1}(\rho)\right)_{\rho=\lambda}+\left(6 \bar{A}+\bar{D} / \lambda^{2}-6 \bar{F} / \lambda^{4}\right)\right], \\
\mathscr{F}_{y}^{\varepsilon}=-\mu \lambda^{2} \int_{0}^{2 \pi}\left(\partial_{\rho}^{3} \delta \Phi_{1}\right)_{\rho=\lambda} \cos \phi d \phi \\
=-\mu \lambda^{2} \pi \delta\left[-4 D / \lambda^{2}\right] .
\end{gathered}
$$

In the case where there are no corrugations $(\varepsilon=0)$, the components become

$$
\begin{aligned}
\mathscr{F}_{x}^{0}= & \frac{\rho_{0}\left(\mathscr{A}_{0}^{\prime}\right)^{2} \pi \delta}{\left(1-\lambda^{2}\right)\left(1-\lambda^{2}+\left(1+\lambda^{2}\right) \log \lambda\right)^{2}}\left[\left(1-\lambda^{4}\right)\left(1+10 \lambda^{2}+\lambda^{4}\right)\right. \\
& \left.+\left(1-\lambda^{2}\right)\left(11+32 \lambda^{2}+5 \lambda^{4}\right) \log \lambda+6\left(1+7 \lambda^{2}+\lambda^{4}-\lambda^{6}\right)(\log \lambda)^{2}\right], \\
\mathscr{F}_{y}^{0}= & -\rho_{0}\left(\frac{\mathscr{A}_{0}^{\prime}}{R}\right) \frac{4 \pi \delta}{1-\lambda^{2}+\left(1+\lambda^{2}\right) \log \lambda} .
\end{aligned}
$$


Here $\rho_{0}$ is the fluid density. Note that these formulas are accurate to $O\left(\delta^{3}\right)$ and reduce, under thin film approximation $(1-\lambda) \ll 1$, to Eqs. (77) and (76) of [2]. With boundary perturbation, $\varepsilon>0$ (with $O^{\prime} \equiv O\left(m \varepsilon, m^{2} \varepsilon\left(1-\lambda^{2}\right)^{-1}\right)$ ),

$$
\begin{aligned}
& \mathscr{F}_{x}^{\varepsilon}=\mathscr{F}_{x}^{0}\left(1-\frac{m \varepsilon}{1-\lambda^{2}}\right)^{-2}\left(1-\frac{m \varepsilon}{1-\lambda^{2}}+O^{\prime}\right), \\
& \mathscr{F}_{y}^{\varepsilon}=\mathscr{F}_{y}^{0}\left(1-\frac{m \varepsilon}{1-\lambda^{2}}\right)^{-1}\left(1-\frac{m \varepsilon}{1-\lambda^{2}} \frac{1-\lambda^{4}+4 \lambda^{2} \log \lambda}{2\left(1-\lambda^{2}+\left(1+\lambda^{2}\right) \log \lambda\right)}+O^{\prime}\right) .
\end{aligned}
$$

Replacing $\lambda^{2}$ by $\lambda^{2}+m \varepsilon$ normalizes the formulas with respect to fluid area. This gives, using this film approximation,

$$
\begin{aligned}
& \mathscr{F}_{x, N}^{\varepsilon}=\rho_{0} \frac{2 \pi}{5} \frac{\Omega^{2} \lambda^{2} \delta}{1-\lambda^{2}}\left(1+2 \frac{m \varepsilon}{1-\lambda^{2}}+O^{\prime}\right), \\
& \mathscr{F}_{y, N}^{\varepsilon}=\rho_{0} 48 \pi \frac{\Omega \lambda^{2} \delta}{R\left(1-\lambda^{2}\right)^{3}}\left(1+6 \frac{m \varepsilon}{1-\lambda^{2}}+O^{\prime}\right) .
\end{aligned}
$$

Finally, note that the torque and angular velocity are

$$
\mathscr{G}_{N}=4 \pi \rho_{0} \frac{\mathscr{A}_{0}}{R}\left(1+\frac{m \varepsilon}{1-\lambda^{2}}+O\left(m \varepsilon, \delta^{2}, R^{2}\right)\right), \quad \partial_{\rho_{\delta}} \psi\left(\rho_{\delta}=\lambda\right)=\Omega \lambda-O\left(\delta^{2}\right) .
$$

Equation (78) of [2] asserts that the torque required to maintain the velocity increases with displacement $\delta$. This extension is not pursued here. It is known that roughness has a retarding effect for the case $\delta=0$ [3]; so it is conjectured that the torque will increase with $m \varepsilon$ along with $\delta^{2}$.

Discussion. A natural parameter is found for the effect of roughness. The boundary perturbations are of height $\varepsilon$, and spaced a distance $2 \pi / n$. It is proposed that a first-order effect of roughness depends on the quantity

$$
m \varepsilon \approx \frac{(\text { roughness height })^{2}}{\text { spacing of peaks }} .
$$

This is supported by the work in [3]. In Sec. 5 it is found that boundary perturbations can induce early separation. Load capacity is effected in the following way. Using the thin film approximation, it is found that (see Fig. 4)

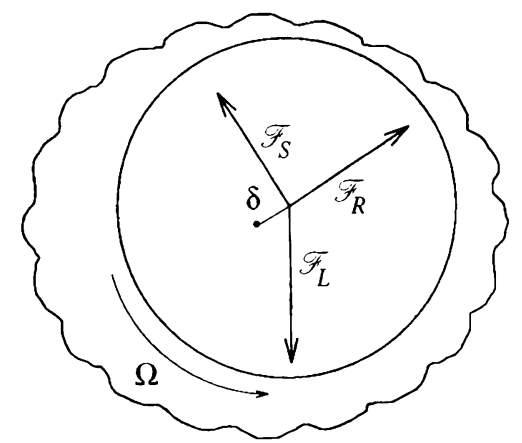

FIG. 4. Force balance diagram with load 


$$
\mathscr{F}_{L}^{\varepsilon}=\mathscr{F}_{L}^{0}\left[1+6 \frac{m \varepsilon}{1-\lambda^{2}}+O(R m \varepsilon)+O^{\prime}\right]
$$

where

$$
\begin{aligned}
\mathscr{F}_{L}^{0} & =\left(\left(\mathscr{F}_{x}^{0}\right)^{2}+\left(\mathscr{F}_{y}^{0}\right)^{2}\right)^{1 / 2} \\
& =\frac{\rho_{0} \mathscr{A}_{0}^{\prime}}{R} \frac{48 \delta \pi}{\left(1-\lambda^{2}\right)^{2}}\left[1+\frac{R^{2} \mathscr{A}_{0}^{\prime 2}}{2} \frac{\left(1-\lambda^{2}\right)^{4}}{(120)^{2}}\left(1+O\left(1-\lambda^{2}\right)\right)+O\left(R^{3}\right)\right] .
\end{aligned}
$$

There is an increase in the load capacity for increasing $R$. As well, for Stokes flow, roughness increases loading ability (also found in the numerical results of [5]).

Finally, for a free rotating cylinder, stability is related to $\mathscr{F}_{x}$. From $(6.5 \mathrm{a})$,

$$
\mathscr{F}_{x}^{\varepsilon} \propto \delta+O\left(\delta^{3}\right)
$$

so if the cylinder is centered at the origin, it is unstable. The presence of roughness $(\varepsilon>0)$ means an increase in the coefficient of proportionality, hence a decrease in stability.

Acknowledgments. The author would like to thank Professors K. B. Ranger and J. B. Keller for many suggestions and references.

Appendix.

$$
\begin{aligned}
& B_{1}(\rho)=\mathscr{A}^{\prime}\left[\frac{5}{8}(A(1-m \varepsilon)-D / 4) p^{3}+\left(A p^{5}\right) / 24-(A(1-m \varepsilon)-D / 4) \frac{p^{3}}{2} \log p\right. \\
& \left.+\frac{1}{4} d(1-m \varepsilon) p(\log p)^{2}+O\left(\rho^{n}\right)\right], \\
& \bar{D}=\mathscr{A}^{\prime 2}\left[1-\frac{2 m \varepsilon}{1-\lambda^{2}}+O\left(m^{2} \varepsilon, m \lambda^{n}\right)\right]\left(1-\lambda^{2}\right) \times \\
& \frac{\left(1+4 \lambda^{2}+\lambda^{4}\right)-\left(1-\lambda^{2}\right)\left(1-26 \lambda^{2}+\lambda^{4}\right) \frac{\log \lambda}{1-\lambda^{2}}-6\left(1-5 \lambda^{2}-\lambda^{4}+\lambda^{6}\right)\left(\frac{\log \lambda}{1-\lambda^{2}}\right)^{2}}{24\left(1-\lambda^{2}+\left(1+\lambda^{2}\right) \log \lambda\right)^{2}} \\
& \frac{2 \bar{A}-2 \bar{F}}{\lambda^{4}}=\frac{\bar{D}}{\lambda^{2}}+\mathscr{A}^{\prime 2} \frac{\lambda^{2}\left(9+4 \lambda^{2}-13 \lambda^{4}\right)+4\left(6-6 \lambda^{2}+8 \lambda^{4}+3 \lambda^{6}\right) \log \lambda-48 \lambda^{2}(\log \lambda)^{2}}{48\left(1-\lambda^{2}\right)\left(1-\lambda^{2}+\left(1+\lambda^{2}\right) \log n \lambda\right)} \\
& \times\left[1-\frac{2 m \varepsilon}{1-\lambda^{2}}+O\left(m^{2} \varepsilon, m \lambda^{n}\right)\right] .
\end{aligned}
$$

\section{REFERENCES}

[1] B. Y. Ballal and R. S. Rivlin, Flow of a Newtonian fluid between eccentric rotating cylinders: Inertial effects, Arch. Rat. Mech. Anal. 62, 237-294 (1976)

[2] R. C. DiPrima and J. T. Stuart, Flow between eccentric rotating cylinders, J. Lubr. Tech. (Trans. ASME), July 1972, pp. 266-274

[3] K. Ma and D. W. Pravica, Separation of streamlines for spatially periodic flow at non-zero Reynolds numbers, ZAMP 43, 1072-1089 (1992)

[4] J. M. Ottino, The kinematics of mixing: Stretching, chaos and transport, Cambridge University Press, Cambridge, 1989 
[5] N. Patir and H. S. Cheng, Application of average flow model to lubrication between rough sliding surfaces, ASME J. Lubr. Tech., Vol. 101, April 1979, pp. 220-230

[6] K. B. Ranger, Separation of streamlines for spatially periodic flow at zero Reynolds numbers, Quart. Appl. Math. 47, 367-373 (1989)

[7] K. B. Ranger, A problem on the slow motion of a viscous fluid between two fixed cylinders, Quart. J. Mech. Appl. Math 14, 411-421 (1961)

[8] G. H. Wannier, A contribution to the hydrodynamics of lubrication, Quart. Appl. Math. 8, 1-32 (1950) 